\title{
El uso del formato audiovisual en Facebook: un medio para representar la identidad
}

\section{The use of the audiovisual format in Facebook: a means for identity representation}

\section{A utilização do formato audiovisual no Facebook: um meio para representar a identidade}

Javier Alejandro Corredor-Aristizábal, Universidad Nacional de Colombia, Bogotá, Colombia (jacorredora@unal.edu.co)

Manuel Alberto Corredor-Aristizábal, Fundación Universitaria Agustiniana Uniagustiniana, Bogotá, Colombia (mcorredor@gmail.com)

RESUMEN | Este artículo describe los objetivos identitarios y los temas que los estudiantes universitarios ( $\mathrm{N}=231$ ) tratan en formato audiovisual en sus muros de Facebook. Para tal fin, se realizó un análisis de contenido de una muestra de 1514 publicaciones de los usuarios. Estas publicaciones fueron codificadas en términos de sus objetivos identitarios, de los temas abordados, de la acción realizada y del formato utilizado, para evaluar si el formato audiovisual tenía objetivos y usos diferentes a los demás usados por los estudiantes en las redes. Los objetivos identitarios se definen como la finalidad de la publicación en relación con la presentación del self en un espacio público. Los resultados muestran que las publicaciones audiovisuales cumplen un rol importante en la construcción de la identidad virtual de los jóvenes. Se utilizan con objetivos identitarios de autodescripción y transmisión de contenidos, y cubren un amplio rango de temas, incluyendo algunos asociados a series y películas.

PALABRAS CLAVE: redes sociales; identidad; formato audiovisual; jóvenes. 
ABSTRACT/This article aims at studying the identity goals and the topics associated to the audiovisual format in social profiles of young users. To this end, a content analysis of 1514 posts was conducted in terms of their topic, their identitary goal, the type of action, and the format used on each of them. This was done to evaluate whether the audiovisual format had different goals and uses than the other formats used in social networks. Identity goals are defined as the purposes of the posts regarding the self-presentation in the public space. Results show that audiovisual posts have an important role in the identity construction process. They are used with identitary goals of self-description and content transmission, and cover a broad range of topics, some of them associated with series and movies.

KEYWORDS: social networks; identity; audiovisual format; youth.

RESUMO|Este artigo descreve os objetivos identitários e os temas que os estudantes universitários ( $\mathrm{N}=231$ ) discutem em formato audiovisual nos seus murais de Facebook. Para isso, foi realizada uma análise de conteúdo de uma amostra de 1514 posts, codificados em termos dos temas e dos objetivos identitários da publicação, assim como da ação realizada e do formato usado. Isto foi feito a fim de avaliar se o formato audiovisual tinha objetivos e usos diferentes dos outros formatos utilizados nas redes sociais. Os objetivos de identidade são definidos como a finalidade da publicação em relação à apresentação do self num espaço público. Os resultados mostram que as publicações audiovisuais têm um papel importante na construção da identidade virtual dos jovens. Estas são utilizadas com objetivos identitários de autodescrição e transmissão de conteúdo, e abrangem temas diversos, incluindo alguns associados a séries e filmes.

PALAVRAS-CHAVE: redes sociais; identidade; formato audiovisual; jovens. 


\section{EL FORMATO AUDIOVISUAL: UN MEDIO PARA REPRESENTAR LA IDENTIDAD EN FACEBOOK}

Durante los últimos años, hemos presenciado una transformación radical en las formas de consumo y producción cultural, que ha sido motivada, en gran medida, por los cambios en el acceso a la información derivados de la aparición de Internet (Corredor, Pinzón, \& Guerrero, 2011). Por esto, la red se ha constituido en un nuevo espacio de acción y construcción identitaria (Alonso, 2015; Manago, Graham, Greenfield, \& Salimkhan, 2008). En las redes sociales, los usuarios han encontrado un espacio para narrarse públicamente y construir complejas descripciones virtuales de sí mismos durante sus procesos de construcción de la identidad. Consistentemente, la investigación previa muestra que existe una relación profunda entre conducta virtual y diversas variables psicológicas (Hughes, Rowe, Batey, \& Lee, 2012; Igartua \& Rodríguez-de-Dios, 2020; Ross, Orr, Sisic, Arseneault, Simmering, \& Orr, 2009; Seidman, 2013).

Este estudio tiene como finalidad determinar, en una muestra de estudiantes universitarios, con qué objetivos usan el formato audiovisual en Facebook y cuál es la relación entre estos objetivos y el proceso de construcción de la identidad. Los objetivos y su relación con la identidad fueron identificados a partir de definiciones previamente establecidas en la literatura, que fueron codificadas mediante un análisis de contenido (Corredor et al., 2011). Este proceso se enfocó en establecer si las publicaciones apuntaban a presentar elementos del self en el espacio público, a identificar cuáles son los contenidos asociados a la identidad que más se presentan en dicho formato, y a evaluar si los objetivos y acciones del formato audiovisual son diferentes de los de otros formatos usados en las redes.

\section{La importancia del desarrollo de la identidad}

La identidad es uno de los conceptos cardinales para comprender el desarrollo adulto y las trayectorias de vida de las personas. Al construir su identidad, las personas asumen ciertos valores, contenidos, roles, prácticas y creencias como elementos fundamentales de la definición de sí mismas (Schoen-Ferreira, AznarFarias, \& Silvares, 2003). En este proceso se establecen los elementos constitutivos del self adulto, un factor determinante para el bienestar subjetivo y la capacidad de resistir presiones sociales de diverso tipo (Martinez \& Dukes, 1997). Tradicionalmente, el proceso de desarrollo de la identidad se realizaba en ambientes offline, como el barrio o el bar. Estos ambientes de interacción informal permitían ensayar diferentes preferencias y aprender a negociarlas con el mundo social, lo que, a su vez, facilitaba procesos de exploración clave para el desarrollo de la identidad (Dunkel, 2000).

De acuerdo con la teoría de la identidad, una persona la desarrolla mediante dos procesos complementarios: la exploración y el compromiso (González, Cuéllar, 
Miguel, \& Serra-Desfilis, 2009; Marcia, 1966). Ambos procesos requieren que las personas presenten sus elecciones en el espacio público (Côté \& Levine, 2014). Por esto, la disponibilidad de espacios para presentar contenidos es fundamental para el desarrollo identitario (Côté, 2006). En este sentido, el desarrollo de la identidad depende de actividades como transmitir contenidos, construir redes interpersonales, la conversación general y la autodescripción. Esto es así porque dichas actividades permiten que las personas presenten públicamente elementos asociados con su identidad y tengan la oportunidad de discutirlos frente a una comunidad de referencia.

\section{Los cambios en el desarrollo de la identidad en el mundo contemporáneo}

Hasta finales del siglo XX, se afirmaba que el proceso de desarrollo identitario se producía durante la adolescencia. Al final de esta, el sujeto estaba listo para asumir los retos de la adultez y enfrentarse a dilemas subsiguientes en el ciclo vital, como la elección de pareja, o de actividades laborales y profesionales (Arnett, 2000). La investigación contemporánea señala que el desarrollo identitario tiene actualmente una trayectoria no lineal y variable en el tiempo, que ha derivado de cambios sociales como la flexibilización laboral, el aumento de las opciones disponibles y el debilitamiento de las restricciones sociales sobre los vínculos románticos (Côté \& Levine, 2014). Estos mismos autores proponen que la identidad adulta, en el mundo contemporáneo, no es un estado final y estático, sino un permanente proceso de negociación frente al ambiente. Esto alarga los periodos de exploración de la identidad. La entrada en la adultez hoy en día sucede después de los 30 años (Arnett, 2000). Este cambio se explica, en parte, por la ampliación de las opciones disponibles, los mundos posibles, a los cuales pueden acceder los jóvenes (Côté, 2006). Si en el siglo pasado las personas debían elegir entre las opciones disponibles en el espacio físico, actualmente pasan por la información disponible en Internet y la aparición de espacios virtuales, en los que es posible desarrollar la identidad (Buckingham, 2008; Corredor et al., 2011). El ejemplo clásico de este tipo de transición es el crecimiento de subculturas juveniles en redes globalizadas, cuyos miembros no tienen contacto físico directo. En el pasado, la interacción con pares, fundamental para evaluar y consolidar las elecciones identitarias, se realizaba primordialmente en el espacio físico. En este contexto, las opciones para la identidad se identificaban en la calle del barrio, el conjunto cerrado o el bar de la esquina, y era allí donde se interactuaba con otros para testearlas (Josselson, 1980). Hoy en el día, el surgimiento de las megaciudades y el aumento del control parental sobre el desarrollo de los adolescentes coinciden con el traslado de estos procesos juveniles a los ambientes sociales digitales. Así, aspectos importantes del sí mismo han comenzado a representarse de manera cada vez más sistemática en los espacios virtuales (Nagy \& Koles, 2014). 


\section{Internet e identidad distribuida en las redes sociales}

El desarrollo de la identidad pasó de ser un proceso exclusivo del mundo offline a ser un proceso de representación que se construye tanto en este último como en los ambientes en línea. Un caso ejemplar de este proceso es el desarrollo de perfiles en las redes sociales virtuales, donde los jóvenes representan sus identidades en términos de sus preferencias personales en diversos niveles (Bozkurt \& $\mathrm{Tu}, 2016$; Zappavigna, 2014). Estas representaciones sirven para articular su vida social y crear representaciones externas de elementos identitarios, lo que constituye un factor protector de la identidad en momentos de crisis (Curwood \& Gibbons, 2010; Halverson, 2005). De hecho, las representaciones virtuales son procesadas como descripciones efectivas de las personas detrás de los perfiles (Farquhar, 2013), y aquellos de las redes sociales son elaborados pensando en una audiencia imaginada receptora de los mensajes (Litt, 2012). Así, los usuarios de estas últimas se involucran en complejos procesos psicológicos para definir las categorías identitarias disponibles en sus perfiles virtuales (Bouvier, 2012). Esto no es exclusivo de los públicos jóvenes; se ha extendido cada vez más a los adultos y a los mayores, quienes utilizan las redes sociales para gestionar crisis identitarias y reconstruir sus preferencias en diversos períodos de transición personal (Castro \& Corredor, 2016; Jung \& Sundar, 2018; Sinclair \& Grieve, 2017). En esta tarea, las personas utilizan la presentación pública de contenidos, en algunos casos audiovisuales, como una herramienta para desarrollar la identidad, lo que implica que, además de autodescripciones, las personas transmiten contenidos durante este proceso (Corredor et al., 2011). Las tecnologías digitales, dadas sus características y posibilidades expresivas, se han convertido en tecnologías de conexión y representación identitaria (Serrano-Puche, 2013).

En las redes sociales digitales, los usuarios no solo actúan como consumidores de contenidos, sino también como productores y distribuidores activos, automotivados por sus propias preferencias identitarias (Martínez-Sala, Segarra-Saavedra, \& Monserrat-Gauchi, 2018).

\section{El formato audiovisual en las redes sociales}

El uso de las redes sociales en el mundo reciente ha estado marcado por dos tendencias de desarrollo de contenidos y de tecnología muy relevantes. Por un lado, la audiovisualidad del lenguaje de la red es creciente (Campos Freire, 2008; Livingstone, 2004). Por otro, los contenidos audiovisuales son predominantes en los mensajes virales en la mayoría de las redes. Adicionalmente, y seguramente de forma complementaria, la distribución de contenidos se ha asociado al procesamiento algorítmico de la información de los usuarios, con el uso de formas de navegación determinadas por el aprendizaje automático que permiten ofrecer a los usuarios contenidos y publicidad vinculada con sus intereses (Napoli, 2014). 
La configuración actual de la tecnología y el ecosistema de la producción y consumo audiovisual implican la convivencia entre contenidos producidos por profesionales de la industria y la generación de contenidos por parte de los prosumidores (Martínez-Sala et al., 2018). Además, aumentan y se diversifican los períodos de consumo audiovisual (que han crecido en cantidad de horas/día per cápita) y se multiplican las opciones de acceso (Hemetsberger, 2003; Winter, 2012). En este contexto, los jóvenes emplean y prefieren el formato audiovisual para desarrollar la identidad, que ha creado un nuevo espacio para la expresión y validación social de elecciones personales. En concordancia con lo anterior, este estudio apunta a responder dos preguntas básicas: ¿Cuáles son los objetivos del uso del formato audiovisual en la presentación de la identidad en redes sociales virtuales? ¿Qué tipos de contenidos identitarios se presentan a través de dicho formato? La hipótesis en relación con la primera pregunta es que los contenidos audiovisuales se relacionan con objetivos identitarios identificados en la literatura previa, tales como la autodescripción, y que este tipo de objetivos son más comunes en los posts en formato audiovisual que en los posts en otros formatos. La hipótesis en relación con la segunda pregunta es que los temas políticos, estéticos, académicos, deportivos, comerciales, laborales y religiosos se asocian con la presentación de la identidad en redes virtuales mediante el formato audiovisual (Corredor et al., 2011).

\section{MÉTODO}

En este estudio se realizó un análisis de contenido de publicaciones en formato audiovisual en muros de Facebook de jóvenes, en función del objetivo, la acción y el tema de ese tipo de posts. A partir de estas codificaciones, se llevaron a cabo correlaciones y análisis factoriales para determinar cómo el formato audiovisual sirve a objetivos identitarios y se usa para transmitir y distribuir ciertos tipos específicos de contenidos, en comparación con los objetivos y usos asignados a otros formatos. Las características de los participantes, la codificación y los instrumentos utilizados se presentan a continuación.

\section{Participantes}

Participaron 231 estudiantes universitarios con una media de edad de 19,6 (D.E.=1,99). De ellos, 119 eran hombres y 112, mujeres. La figura 1 muestra su frecuencia de edades, de los 16 a los 24 años. Los participantes fueron contactados a través de convocatorias abiertas en diferentes universidades de Bogotá, por medio de correos electrónicos directos, invitándolos a participar en un estudio sobre el comportamiento en redes sociales virtuales. Estas listas son de acceso público y son frecuentemente utilizadas por estudiantes y profesores para pedir ayuda o difundir información. La participación fue voluntaria y se clasificó como una observación 


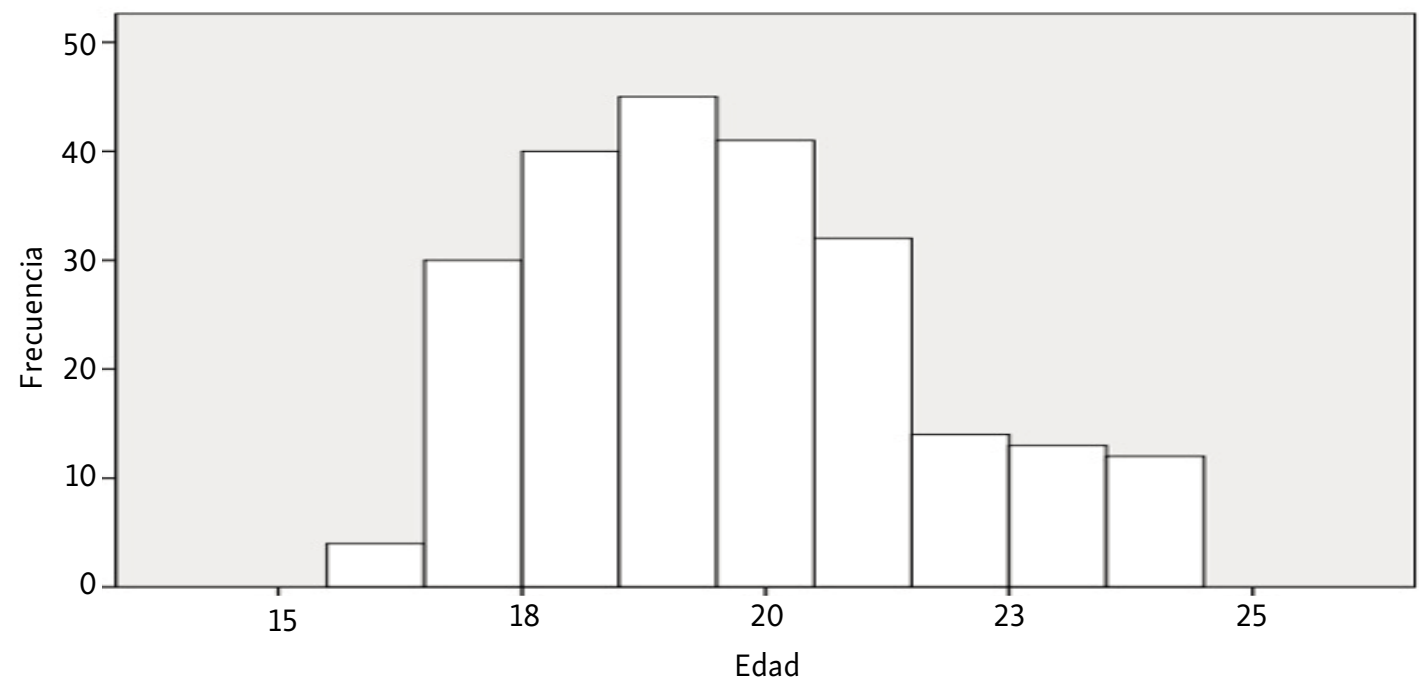

Media $=19,64 ;$ D.E. $=1,98 ; \mathrm{N}=231$.

Figura 1. Distribución de la muestra por edades

Fuente: Elaboración propia.

naturalista en un contexto virtual, sin probabilidad de daño, y con almacenamiento de datos anónimos como es permitido por los estándares éticos de la investigación en psicología (American Psychological Association, 2017). De acuerdo con aquellos requerimientos, se obtuvo el consentimiento informado al comienzo de la aplicación del formulario virtual. En el caso de menores de edad, se solicitó el envío de un formulario de consentimiento firmado por sus padres o cuidadores.

A quienes respondieron señalando querer participar, se les pidió agregar como amigo un perfil de Facebook creado para este estudio. Adicionalmente, se les solicitó responder un formulario virtual sobre características sociodemográficas (edad, género, carrera universitaria) y ciertos patrones de comportamiento virtual (frecuencia de uso, privacidad del perfil). Después de copiar la actividad de los usuarios, se les desagregó como amigos de la página de perfil utilizada para este estudio, para mantener la confidencialidad de acciones posteriores.

Los participantes estaban matriculados en 62 carreras universitarias de 13 universidades colombianas y llevaban entre uno y 10 semestres de estudio. Todos quienes decidieron participar fueron incluidos en este estudio y reportaron tener una actividad frecuente en redes sociales. Entraban por lo menos una vez al día a sus cuentas de Facebook, de acuerdo con la siguiente distribución diaria: 33,5\% más de 3 horas; $23 \%$ entre 2 y 3 horas; $25,2 \%$ entre 1 y 2 horas, y $18,2 \%$, menos de una hora. Las carreras de los estudiantes cubrían un amplio rango, incluyendo ciencias sociales, ingenierías, ciencias de la salud y áreas básicas. 


\section{Datos}

Los muros fueron recolectados durante el segundo semestre del año 2017 y los datos codificados en 2018. La muestra cubría un rango de tres días (viernes a domingo). Este rango fue elegido para tener un muestreo de la conducta virtual durante un fin de semana, cuando hay un mayor número de interacciones en esta red social (Pempek, Yermolayeva, \& Calvert, 2009). La mayoría de las obligaciones académicas y laborales suceden de lunes a viernes, y el fin de semana permite un uso más flexible del tiempo. El fin de semana observado no fue el mismo para todos los participantes: se copiaron las publicaciones realizadas en sus muros durante el fin de semana anterior a la inscripción al estudio; al dar acceso a sus perfiles al inscribirse en el estudio, se podían ver sus publicaciones previas. Como los estudiantes se inscribieron en diferentes oleadas, los tres días observados no correspondieron a las mismas fechas para todos, sino que se distribuyeron a lo largo de cuatro fines de semana. No se emplearon perfiles abiertos para tener un acceso directo a los contenidos que las personas deciden presentar a sus comunidades de referencia. Esto se confirmó con el formulario sociodemográfico. Cuando se preguntó a los participantes si la configuración de privacidad permitía el acceso completo de los investigadores a los contenidos del perfil, 92,1\% respondió afirmativamente y 4,8\% contestó que no conocía cuál era la configuración de privacidad de su perfil.

Se eligió Facebook pues es una red que permite diferentes tipos de interacción (texto, video, fotos), porque la interacción y el posteo son públicos, y porque no existen límites en las longitudes de las publicaciones, lo que permite realizar comparaciones entre el uso de lo audiovisual y otros formatos de presentación de contenidos. En redes cuyo contenido es primordialmente audiovisual, como Instagram, o enfocadas en lo escrito y con restricciones de espacio, como Twitter, el formato del contenido sería relativamente homogéneo. Estas características permiten una interacción compleja en comunidades relativamente permanentes de usuarios, que actúan como pares para validar y testear elecciones identitarias. En la elección influyó también el hecho de que existen precedentes de investigación en Latinoamérica que indican una relación entre el comportamiento en esta red y el desarrollo identitario (Corredor et al., 2011). Adicionalmente, los niveles de penetración de Facebook son los más altos entre todas las redes (Almansa-Martinez, Fonseca, \& Castillo-Esparcia, 2013; Carmona, 2011; Krallman, Pelletier, \& Adams, 2016).

Los muros de los usuarios fueron copiados en archivos fijos para ser posteriormente codificados. Específicamente, se guardó una captura de pantalla en un archivo Excel, que preservaba los títulos de los contenidos de las publicaciones y la descripción realizada por Facebook (por ejemplo, "X publicó un video"). Este procedimiento permitió obtener una muestra de 1514 posts, en la cual $96 \%$ de los participantes presentó algún tipo de actividad. Se decidió copiar y analizar solo 
un fin de semana, porque se intentaba obtener una muestra del comportamiento virtual típico de un estudiante universitario durante este período, durante un fin de semana del año. En el semestre previo al inicio de la investigación se indagó mediante una encuesta corta las condiciones en las que se sentirían más dispuestos a dar acceso a sus perfiles y a compartir sus muros. Se encontró que preferían limitar la copia del muro a un período restringido y así se planteó en el consentimiento informado. En este proceso también se recolectaron fragmentos de muros para revisar la pertinencia de las categorías establecidas en la literatura previa sobre objetivos identitarios.

El corpus de este estudio cubría cuatro fines de semana, en uno de los cuales se ubicaba el fin de semana observado para cada participante. Este diseño permitía, dado el tamaño de la muestra, que eventos puntuales en el comportamiento de los diferentes usuarios se equilibraran a lo largo de la muestra. Si todos los datos correspondieran exactamente a la misma fecha, eventos compartidos (como un día feriado) podrían afectar las observaciones. Lo mismo sucedería si un usuario tuvo un evento importante durante el período observado (por ejemplo, un cumpleaños). Estos problemas se evitaron al tener una muestra distribuida en cuatro fines de semana. Después de que los estudiantes respondieron a la invitación y a los cuestionarios demográficos mencionados con anterioridad, se realizó una copia de las publicaciones realizadas entre el viernes y el domingo anteriores a dicha fecha. La recolección se llevó a cabo durante 2017. Aunque puede haber habido una evolución en el comportamiento en Facebook con posterioridad a esta fecha, es posible especular que la primacía de lo audiovisual en el comportamiento del usuario no parece haber disminuido sino, por el contrario, aumentado. El crecimiento de redes como Instagram y Tik-Tok son prueba de ello.

\section{Análisis de contenido}

Para caracterizar el comportamiento virtual de los usuarios, se llevó a cabo un análisis de contenido, una codificación estructurada de tipo mixto, en la que se incluyen categorías establecidas a priori, a partir de la revisión teórica y emergentes identificadas en los datos (Namey, Guest, Thairu, \& Johnson, 2007). Las publicaciones fueron codificadas en términos de su objetivo, la acción realizada, el tema y el formato del contenido. Para este proceso se estableció una serie de preguntas (por ejemplo, ¿qué objetivo?, ¿qué acción?) y una serie de categorías posibles (como la transmisión de contenidos como objetivo) a partir de la literatura previa (Corredor et al., 2013; Corredor et al., 2011). Las categorías fueron refinadas a partir de la codificación de un segmento parcial de los muros (10\%), y se incluyeron nuevas categorías de respuesta cuando se estableció que la codificación existente no cubría aspectos importantes de los datos. Este proceso fue realizado por los mismos codificadores a cargo de la codificación final (ver sección de confiabilidad). 
En términos del objetivo de la publicación, se codificaron como transmisión de contenidos aquellas publicaciones en las que el contenido textual del post no era creado por el usuario. Esta categoría podría incluir hipervínculos o fotografías y se codificaba cuando el post no iniciaba una conversación, no usaba expresiones emocionales dirigidas a otros usuarios específicos, ni frases en primera o en tercera persona. Se codificaron como publicaciones de objetivo interpersonal aquellas en las que el usuario realizaba expresiones de afecto hacía otros usuarios en su red, no había personajes famosos, grupos musicales o similares. Se codificaron como publicaciones de autodescripción aquellas en las que el usuario expresaba en primera persona (con la inclusión de yo o mediante la conjugación verbal), su opinión sobre un contenido (creencia, etc.) o realizaba una descripción de sí mismo. También se incluyeron en esta categoría selfies, fotos del usuario, de perfil o de portada. Se codificaron como publicaciones de conversación general aquellas en las que la persona realizaba conversaciones de diversos tipos, con o sin fotos, sin hipervínculos y con comentarios. Si la publicación incluía elementos de otras categorías, pero estaba en el contexto de una conversación, se codificaba como conversación general. Estas categorías se relacionan con el desarrollo de la identidad, porque la presentación de elecciones personales y la autodescripción en un contexto público de validación son centrales a la construcción de la identidad (Bozkurt \& Tu, 2016; Corredor et al., 2011; Zappavigna, 2014). De hecho, la literatura previa considera que son objetivos identitarios el mantenimiento de una red social mediante acciones interpersonales, la conversación general, y la presentación de elecciones personales a través de la transmisión de contenidos (Dunkel, 2000; Litt, 2012).

En términos de la acción realizada, se consideraron categorías generales que describían las actividades que podían realizar los usuarios dentro de la red. En este caso, se utilizaron como categorías subir un hipervínculo con contenido escrito, musical o audiovisual, con comentario o sin comentario adicional; escribir una publicación propia en la que participante expresara una opinión o idea de manera escrita en su propio muro; comentar un post en el muro de otra persona; usar expresiones emocionales dentro del ambiente virtual (como $(-)$, dar "me gusta"); subir una foto, y agregar un amigo o grupo dentro de la red. Estas definiciones, dada su naturaleza operacional, permitían capturar la conducta virtual de los participantes de una manera directa.

En términos del formato de la publicación, este se consideraba escrito cuando solo tenía texto producido por el participante o por otros; se consideraba audiovisual cuando presentaba contenidos que incluían audio y video simultáneamente, y como otro cuando presentaba contenidos que no entraban en las categorías anteriores (como pinturas o memes). Adicionalmente, las publicaciones se codificaron en 
términos de los temas que presentaban: políticos, estéticos, académicos, deportivos, comerciales o laborales, religiosos, personales, humor, autoayuda, videojuegos, culinarios, tecnológicos, salud y belleza, y otros.

\section{Confiabilidad}

Siguiendo los criterios de Queluz, Campos, Santis, Isaac y Barham (2019), la confiabilidad fue evaluada sobre un $19 \%$ de los datos (44 muros), categorizados por cinco codificadores independientes organizados en parejas. Específicamente, las parejas fueron organizadas intercalando los muros que los diferentes miembros del equipo debían codificar. Los codificadores 1 y 2,2 y 3,3 y 4 , y 4 y 5 debieron codificar simultáneamente 11 de los 44 muros mencionados anteriormente. Dado que los codificadores proporcionaban el total para cada categoría dentro de cada muro, se evaluó qué tan similares eran estos totales usando una correlación de Spearman (tabla 1). En todos los casos menos uno, las correlaciones fueron significativas y superiores a ,69. En el caso de agregar un amigo o grupo, la correlación no fue significativa. En este caso, el grado de acuerdo se revisó con tablas de contingencia y la coincidencia fue de $93 \%$. La confiabilidad de los temas se realizó de manera similar. En siete había correlaciones significativas y en tres, correlaciones perfectas. En los cuatro casos en los que no se presentó una correlación significativa, se chequeó la confiabilidad con tablas de contingencias. El porcentaje de acuerdo entre codificadores fue de $65 \%$ para temas académicos, $93 \%$ para comerciales y laborales, $95 \%$ para temas de salud y $60 \%$ para otros.

\section{Análisis de datos}

Se realizaron análisis descriptivos para caracterizar el comportamiento de los participantes en sus redes sociales virtuales y determinar qué objetivos, acciones, formatos y temas eran más comunes. Las diferencias en estas descriptivas fueron evaluadas con ANOVA'sde medidas repetidas. En segundo lugar, se realizaron correlaciones para determinar qué tipos de objetivos identitarios se relacionan con el uso del formato audiovisual. Finalmente, se realizó un análisis factorial de componentes principales para determinar cómo se organizaban los objetivos identitarios y los formatos, tanto a nivel de factores como de la solución gráfica, de este tipo de procedimiento.

\section{RESULTADOS}

Aunque la muestra aquí analizada representa el comportamiento de los usuarios durante un fin de semana y no es generalizable a un período más largo, una descripción general permite dar cuenta de los principales objetivos, acciones, formatos y temas que se asocian al uso del formato audiovisual en la presentación de la identidad virtual de los usuarios durante este rango de tiempo (tabla 1). 


\begin{tabular}{ccccccc} 
& N & Mín. & Máx. & Media & D. E. & Confiabilidad \\
\cline { 2 - 6 } Objetivo & & & & & & \\
\hline Transmisión & 231 &, 0 & 64,0 & 3,05 & 5,81 &, $91^{* *}$ \\
\hline Interpersonal & 231 &, 0 & 18,0 & 1,10 & 2,11 &, $82^{* *}$ \\
\hline Autodescripción & 231 &, 0 & 9,0 &, 80 & 1,24 &, $80^{* *}$ \\
\hline General & 231 &, 0 & 27,0 & 1,59 & 3,39 &, $69^{* *}$ \\
Acción & & & & & & \\
\hline Hipervínculo & 231 &, 0 & 22,0 & 1,51 & 2,46 &, $86^{* *}$ \\
\hline Post & 231 &, 0 & 8,0 &, 97 & 1,53 &, $94^{* *}$ \\
\hline Comentar & 231 &, 0 & 17,0 & 1,12 & 2,06 &, $96^{* *}$ \\
\hline Emocional & 231 &, 0 & 11,0 &, 64 & 1,46 &, $74^{* *}$ \\
\hline Foto & 231 &, 0 & 57,0 & 2,15 & 5,02 &, $90^{* *}$ \\
\hline Amigo/grupo & 231 &, 0 & 1,0 &, 03 &, 17 &,- 033 \\
\hline Formato & & & & & &, $89^{* *}$ \\
\hline Escrito & 231 &, 0 & 16,0 &, 44 & 1,55 &, $71^{* *}$ \\
\hline Audiovisual & 231 &, 0 & 71,0 & 1,47 & 4,92 &, 53 \\
\hline Otro & 231 &, 0 & 3,0 &, 21 & & \\
\hline
\end{tabular}

a Correlación de Spearman ${ }^{*} p<, 01$.

Tabla 1. Descriptivas. Objetivo, acción y formato

Fuente: Elaboración propia.

Las diferencias entre los diferentes objetivos identitarios $(F(3,690)=22,75, p=, 000)$, el tipo de acción $(F=(5,1150)=23,17, p=, 000)$ y el tipo de formato $(F(2,460)=11,90$, $\mathrm{p}=, 000$ ) fueron significativas. Estos resultados indican que la transmisión de contenidos audiovisuales constituye un componente importante de la actividad de los usuarios en redes virtuales. De hecho, en un rango de tres días, los participantes en este estudio compartieron en promedio un video y medio, para un total de 340 posts en formato audiovisual. Dado que la presentación de contenidos en los perfiles de redes virtuales es un acto identitario (Corredor et al., 2011; Manago et al., 2008), la frecuencia de uso del formato audiovisual indica que, al menos en el rango temporal de este estudio, los usuarios utilizan este formato para construir su identidad virtual. 


\begin{tabular}{ccccccc} 
& N & Mín. & Máx. & Media & D. E. & Confiabilidada \\
\hline Políticos & 231 &, 0 & 10,0 &, 43 & 1,14 &, $77^{* *}$ \\
\hline Estéticos & 231 &, 0 & 10,0 & 1,15 & 1,70 &, $71^{* *}$ \\
\hline Académicos & 231 &, 0 & 12,0 &, 38 & 1,20 &, 27 \\
\hline Deportivos & 231 &, 0 & 4,0 &, 10 &, 43 &, $64^{* *}$ \\
\hline Comercial/laboral & 231 &, 0 & 4,0 &, 11 &, 48 &,- 034 \\
\hline Religión & 231 &, 0 & 2,5 &, 04 &, 23 & $1,00^{* *}$ \\
\hline Personal & 231 &, 0 & 66,0 & 4,53 & 6,63 &, $57^{* *}$ \\
\hline Humor & 231 &, 0 & 101,0 & 1,51 & 6,84 &, $60^{* *}$ \\
\hline Autoayuda & 231 &, 0 & 6,5 &, 25 &, 76 &, $92^{* *}$ \\
\hline Videojuegos & 231 &, 0 & 1,5 &, 07 &, 27 &, $85^{* *}$ \\
\hline Culinarios & 231 & 0 & 1 &, 00 &, 07 & $1,00^{* *}$ \\
\hline Tecnología & 231 &, 0 &, 5 &, 00 &, 03 & $1,00^{* *}$ \\
\hline Salud & 231 &, 0 & 1,0 &, 01 &, 10 &,- 024 \\
\hline Otros & 231 &, 0 & 25,0 &, 93 & 2,60 &, 119 \\
\hline
\end{tabular}

a Correlación de Spearman.

Tabla 2. Descriptivas. Temas, publicaciones

Fuente: Elaboración propia.

Cuando se analizaron exclusivamente los contenidos de los hipervínculos presentados por los participantes, se encontró que los temas de contenido personal eran los más frecuentes durante el fin de semana observado (tabla 2). En esa lista, los siguientes temas más frecuentes fueron temas de humor (como memes) y estéticos (por ejemplo, videos musicales). Los siguientes temas incluyeron temas políticos, académicos, de autoayuda y de otra naturaleza. Cuando estas diferencias se compararon usando un ANOVA de medidas repetidas, se encontró que eran significativas $(F(13,2990)=47,295, p=, 000)$. Estas diferencias indican que, temáticamente, los usuarios de la muestra presentan sus elecciones identitarias en sus perfiles virtuales no solo como descripciones personales, sino con opiniones y contenidos, lo que es consistente con la idea discutida en el marco teórico de que la identidad virtual es fundamentalmente performativa y no autodescriptiva (Farquhar, 2013). En otras palabras, la tabla 2 muestra que los usuarios presentan su identidad no solo como una descripción explícita de sí mismos, sino mediante posts cuyo foco temático refleja sus intereses. 


\section{Correlaciones entre objetivos identitarios y uso del formato audiovisual}

En conjunto, los resultados indican que el uso del formato audiovisual correlaciona significativamente con la autodescripción y, en un menor grado, con la transmisión de contenidos. Cuando se realizaron correlaciones entre los formatos y los objetivos de las acciones, se encontraron patrones que arrojan luz sobre los roles del audiovisual en el proceso de la construcción de la identidad virtual (tabla 3). El formato escrito tiene sus mayores correlaciones con acciones cuyo objetivo es la transmisión de contenidos, el desarrollo de relaciones interpersonales, y la conversación general, mientras que el formato audiovisual tiene correlaciones fuertes con la transmisión de contenidos y la autodescripción, y débiles con la conversación general y el mantenimiento de relaciones interpersonales. Para confirmar este resultado, se utilizaron correlaciones parciales, una técnica que permite aislar la relación exclusiva entre dos variables (como autodescripción $\mathrm{y}$ formato audiovisual), controlando por los efectos asociados a otras variables (transmisión de contenidos, conversación general). En este caso, se llevaron a cabo correlaciones parciales en las que se evaluaba la asociación entre un objetivo identitario y el formato audiovisual, controlando por los demás objetivos identitarios. Con este control, se encontró que solo existían correlaciones significativas entre el uso del formato audiovisual y objetivos de autodescripción, y entre este mismo formato $(\mathrm{r}=, 446, \mathrm{p}<, 000)$ y objetivos de transmisión de contenidos $(\mathrm{r}=, 172, \mathrm{p}<, 009)$. Asimismo, cuando se realizó una regresión por pasos incluyendo todos los objetivos identitarios como predictores del uso del formato audiovisual, los únicos objetivos que se mantuvieron dentro del modelo fueron los de autodescripción y los de transmisión de contenidos. Este modelo fue significativo $(F(1,228)=65,74$, $\mathrm{p}<, 000$ ). Esto implica que el formato audiovisual, a diferencia del escrito, se utiliza principalmente para autodescribir la identidad en el contexto virtual y transmitir elecciones identitarias.

Estos resultados permiten señalar que el formato audiovisual se ha constituido efectivamente en un medio para la autodescripción, es decir, para la expresión identitaria. Este resultado extiende la literatura previa que señalaba que las redes sociales permiten el surgimiento de una identidad distribuida (Corredor et al., 2011; Farquhar, 2013), para especificar que dicha identidad tiene fuertes componentes audiovisuales. En este contexto, es importante tener claro que la asociación de la identidad pública a objetos culturales en formato audiovisual (como películas, videos musicales) era posible antes de la aparición de las redes sociales, por ejemplo, a través de la opinión en formato oral. Sin embargo, la aparición de las redes sociales virtuales permite asociar directamente contenidos en dicho formato con la identidad distribuida presentada en los ambientes virtuales. 


\begin{tabular}{|c|c|c|c|c|c|c|c|}
\hline & 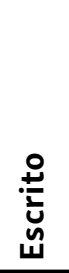 & $\begin{array}{l}\bar{\pi} \\
\frac{\pi}{3} \\
\frac{n}{2} \\
\frac{0}{\overline{0}} \\
\frac{2}{4}\end{array}$ & 온 & 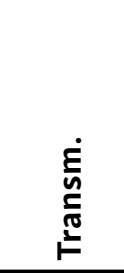 & 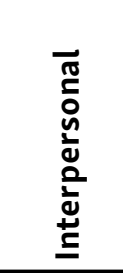 & 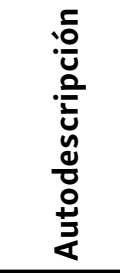 & $\begin{array}{l}\bar{\pi} \\
\frac{0}{0} \\
\frac{c}{0} \\
0\end{array}$ \\
\hline Escrito & 1 & ,075 & ,153* &, $300 * *$ &, $323^{* *}$ & ,079 & $348^{* *}$ \\
\hline Audiovisual & & 1 & ,040 &, $257^{* *}$ &, $154^{*}$ &, $472^{* *}$ &, $187^{* *}$ \\
\hline Otro & & & 1 &, $204^{* *}$ & 128 & ,089 & ,149* \\
\hline Transmisión & & & & 1 &, $548^{* *}$ &, $142^{*}$ &, $258^{* *}$ \\
\hline Interpersonal & & & & & 1 & ,056 &, $268^{* *}$ \\
\hline Autodescripción & & & & & & 1 &, $411^{* *}$ \\
\hline General & & & & & & & 1 \\
\hline
\end{tabular}

${ }^{*}$.Significativa al 0,$05 ; * *$ Significativa al 0,01 .

Tabla 3. Correlaciones entre el contenido y el objetivo identitario de la acción

Para confirmar este resultado, se realizó un análisis factorial de componentes principales dirigido a encontrar patrones de organización en múltiples variables (Alcántar, Valdés, Carlos, Martínez-Ferrer, \& García, 2018; Gálvez-Nieto, VeraBachmann, Trizano-Hermosilla, Polanco, \& Salvo, 2018). Este confirmó que el uso de contenidos audiovisuales se asocia fuertemente con la autodescripción, mientras que los contenidos escritos y de otro tipo se relacionan con otros objetivos (en la figura 2 se puede ver la solución gráfica). El objetivo de transmisión de contenidos no apareció asociado al formato audiovisual en este análisis, probablemente porque la transmisión de contenidos tiene asociaciones más fuertes con el formato escrito (tabla 3). Esto puede hacer que, como se observa en la solución gráfica (figura 2), la transmisión de contenidos aparezca más cerca del formato escrito que del formato audiovisual.

Estos resultados fueron obtenidos tanto para soluciones rotadas con el método Varimax como para soluciones no rotadas que son más resistentes a menores tamaños muestrales (Gouveia, de Moura, de Oliveira Santos, do Nascimento, de Oliveira Guedes, \& Gouveia, 2018; Nunes \& Mota, 2018). Específicamente, el análisis identificó dos factores con valores propios (eigenvalues) superiores a 1. El primer factor explicaba 34\% de la varianza y en él se ubicaban los objetivos de transmisión de contenidos, interpersonal y conversación general, y los formatos escritos y otros. 


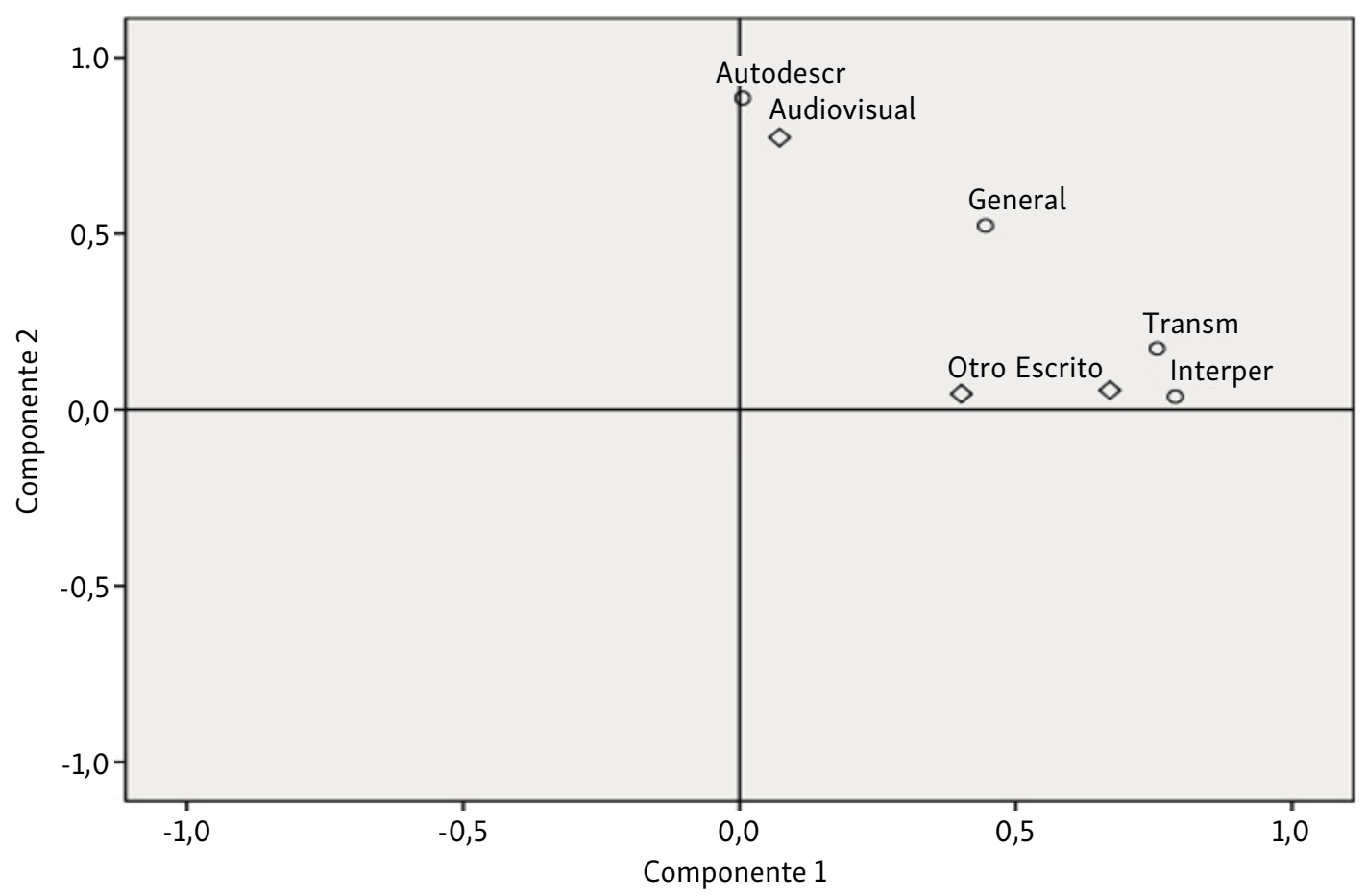

Figura 2. Representación gráfica de la relación entre los tipos de contenido y los objetivos de las acciones en el mundo virtual

Fuente: Elaboración propia.

El segundo factor explicaba $18,8 \%$ de la varianza y en él se ubicaban el objetivo de autodescripción y el formato audiovisual. Es posible interpretar estos dos factores en términos de dos usos de las redes sociales: el primero, asociado a la interacción en formato escrito y el segundo, a la autodescripción de la identidad mediante publicaciones en formato audiovisual. Tanto los análisis de correlaciones (tabla 3) como el análisis factorial (figura 2) señalan que las personas que realizan una mayor cantidad de publicaciones en formato audiovisual también hacen una mayor cantidad de autodescripciones, mientras que las que utilizan el formato escrito lo hacen con otros objetivos identitarios.

\section{CONCLUSIONES}

Este estudio muestra que el uso del formato audiovisual correlaciona fuertemente con la autodescripción y la transmisión de contenidos y con la presentación de temas políticos, estéticos, comerciales, laborales y personales. Este resultado confirma la idea de que los ambientes virtuales han transformado los procesos de construcción de la identidad de una manera que permite la exploración y presentación de opciones y elecciones en ambientes distribuidos (Bozkurt \& Tu, 2016; Corredor et al., 2011; Manago et al., 2008; Zappavigna, 2014). Este estudio amplía además esta idea, al mostrar que el formato audiovisual se constituye en 
un elemento fundamental de dicho proceso. Se podría incluso decir que Facebook ha permitido el surgimiento de una identidad anclada audiovisualmente. Esto no es extraño si se considera que la construcción de la identidad sucede en respuesta a las opciones disponibles en el ambiente social (Côté \& Levine, 2014). En ese sentido, la existencia de perfiles virtuales y la conexión de estos con elementos audiovisuales es una característica de la época.

La literatura previa señala la existencia de identidades distribuidas de modalidad hibrida o blended, que combinan elementos intrapsicológicos con huellas virtuales desplegadas en internet (Corredor et al., 2011). El hecho de que estas huellas utilicen formatos audiovisuales es importante, porque indica que los sujetos se adaptan a los formatos disponibles para expresar sus preferencias e intereses. Esta negociación entre lo que se quiere presentar como parte de la identidad y las posibilidades expresivas del medio ha sido explorada previamente en relación con la producción de arte digital (Halverson, 2010). Al considerarse en relación con el desarrollo de la identidad virtual, este resultado señala que las posibilidades de construcción del sí mismo son amplificadas y modeladas por las características de los medios disponibles en Internet, lo que permite construirse, crear y recrearse a sí mismo mediante el formato audiovisual.

Este estudio tiene varias limitaciones. La primera es la característica del muestreo. Dado que solo se analizó la conducta virtual durante un fin de semana, es difícil estar seguros de que los patrones aquí observados se repetirían exactamente en un tiempo más largo. En este sentido, es importante tener en mente que se analizaron 1514 posts de los cuales solo 340 estaban en formato audiovisual, lo que hace necesario evaluar las relaciones encontradas utilizando técnicas de minería de datos que permitan trabajar con una muestra mayor. La segunda limitación es el hecho de que los cambios en los algoritmos de Facebook pueden haber modificado el comportamiento de los usuarios en dicha red después de la realización de este estudio. Esto es así porque el rol de lo audiovisual en la construcción de la identidad virtual depende de las decisiones que Facebook tome en relación con las opciones de publicación de los usuarios. En otras palabras, es importante recordar que el comportamiento observado es el resultado de la interacción entre las preferencias de los usuarios y las potencialidades de uso (affordances) del medio. En esta misma línea, una limitación adicional es que explora la construcción de la identidad virtual en Facebook, dejando fuera otras redes de creciente penetración como Instagram, Tik-Tok y Twitter. En este sentido, este estudio no da luces sobre el comportamiento en redes diferentes a Facebook, hacia las cuales pueden estar migrando los usuarios. Futuros estudios necesitan evaluar el rol de lo audiovisual en la construcción de la identidad en aquellas para confirmar si la construcción de la identidad virtual también pasa en esos casos por un fuerte uso del formato audiovisual. 


\section{REFERENCIAS}

Alcántar, C., Valdés, A., Carlos, E., Martínez-Ferrer, B., \& García, F. (2018). Propiedades psicométricas de la adaptación al español de la participant role approach (pra) (Psychometric Properties of the Adaptation to Spanish of the Participant Role Approach (PRA)). Revista Colombiana de Psicología, 27(2), 177-192. https://doi.org/10.15446/rcp.v27n2.68721

Almansa-Martinez, A., Fonseca, O., \& Castillo-Esparcia, A. (2013). Social networks and young people. Comparative study of facebook between Colombia and Spain. Comunicar: Revista Científica de Comunicación y Educación, 20(40), 127-135. https://doi.org/10.3916/C40-2013-03-03

Alonso, B. P. (2015). ¿Mi perfil soy yo?: Acercamiento a las concepciones sobre la construcción de identidad en los servicios de redes sociales en internet (My profile defines me? Approach to conceptions of identity construction in social network sites). Revista Cubana de Información $y$ Comunicación, 4(7), 111-139. Retrieved from http://www.alcance.uh.cu/index.php/RCIC/article/view/48

American Psychological Association. (2017). Ethical principles of psychologists and code of conduct. American Psychologist, 57(12), 1060-1073. https://doi.org/10.1037/0003-066X.57.12.1060

Arnett, J.J. (2000). Emerging adulthood: A theory of development from the late teens through the twenties. American Psychologist, 55(5), 469-480. https://doi.org/10.1037/0003-066X.55.5.469

Bouvier, G. (2012). How Facebook users select identity categories for self-presentation. Journal of Multicultural Discourses, 7(1), 37-57. https://doi.org/10.1080/17447143.2011.652781

Bozkurt, A. \& Tu, C. H. (2016). Digital identity formation: socially being real and present on digital networks. Educational Media International, 53(3), 153-167. https://doi.org/10.1080/09523987.2016.1236885

Buckingham, D. (2008). Youth, identity, and digital media. Cambridge, MA: MIT Press.

Campos Freire, F. (2008). Las redes sociales trastocan los modelos de los medios de comunicación tradicionales (The impacts of post-media networks on the traditional media). Revista Latina de Comunicación Social, 11(63), 277-286. Retrieved from http://www.revistalatinacs.org/_2008/23_34_Santiago/Francisco_Campos.html

Carmona, O. I. (2011). 2010: Principales estadísticas sociodemográficas de Internet y Facebook (Main sociodemographic statistics of the Internet and Facebook). Revista ComHumanitas, 2 (1), 97-104. Retrieved from http://www.comhumanitas.org/index.php/comhumanitas/article/view/18

Castro, J. C. \& Corredor J. (2016). Interacción de adultos mayores en redes sociales virtuales (facebook) y su relación con el bienestar subjetivo (Interaction of older adults in virtual social networks (facebook) and its relationship with subjective well-being). Revista Iberoamericana de Psicología: Ciencia y Tecnología, 9(2), 61-71. Retrieved from https://reviberopsicologia.ibero.edu.co/article/view/rip.9205

Corredor, J., Pico, J., Castro, C., Hernández, M., Arias, C., Alviar, C., Rojas, L., Silva, J., \& Niño, J. (2013). La Frontera digital: Efectos psicológicos de Internet en la región de la Orinoquía (The Digital Frontier: Psychological Effects of the Internet in the Orinoquía Region). Revista Iberoamericana de Psicología, 6(2), 55-68. https://doi.org/10.33881/2027-1786.rip.6206

Corredor, J., Pinzón, O., \& Guerrero, M. (2011). Mundo sin centro: Cultura, construcción de la identidad y cognición en la era digital (A World without Center: Culture, Identity Construction, and Cognition in the Digital Age). Revista de Estudios Sociales, (40), 44-56. https://doi.org/10.7440/res40.2011.05 
Côté, J. (2006). Identity studies: How close are we to developing a social science of identity?An appraisal of the field. Identity, 6(1), 3-25. https://doi.org/10.1207/s1532706xid0601_2

Côté, J. E. \& Levine, C. G. (2014). Identity, formation, agency, and culture: A social psychological synthesis. Mahwah, NJ: Psychology Press.

Curwood, J. \& Gibbons, D. (2010). “Just like I have felt": Multimodal counternarratives in youth-produced digital media. International Journal of Learning and Media, 1(4), 59-78. https://doi.org/10.1162/ijlm_a_00034

Dunkel, C. S. (2000). Possible selves as a mechanism for identity exploration. Journal of Adolescence, 23(5), 519-529. https://doi.org/10.1006/jado.2000.0340

Farquhar, L. (2013). Performing and interpreting identity through Facebook imagery. Convergence, 19(4), 446-471. https://doi.org/10.1177/1354856512459838

Gálvez-Nieto, J. L., Vera-Bachmann, D., Trizano-Hermosilla, I., Polanco, K., \& Salvo, S. (2018). Propiedades psicométricas de la versión reducida de la Escala de Valores para el Desarrollo Positivo Adolescente (evdpa-r) en estudiantes chilenos (Psychometric Properties of the Reduced Version of the Positive Adolescent Development Value Scale (EVDPA-R) in Chilean Students). Revista Colombiana de Psicología, 27(2), 69-84. https://doi.org/10.15446/rcp.v27n2.65500

González, J. J. Z., Cuéllar, A. I., Miguel, J. M., \& Serra-Desfilis, E. (2009). El desarrollo de la identidad en la adolescencia y adultez emergente: Una comparación de la identidad global frente a la identidad en dominios específicos (Identity development in the adolescence and emergent adulthood: a comparison of global identity versus identity in specific domains). Anales de Psicología, 25(2), 316-329. Retrieved from https://revistas.um.es/analesps/article/view/87931

Gouveia, V. V., Moura, H. M. de, Santos, L. C. de O., Nascimento, A. M. do, Guedes, I.de O., \& Gouveia, R. S. V. (2018). Escala de Autorrelato de Trapaça-Admissão: Evidências de Validade Fatorial e Precisão (Cheating Admission Self Report Scale: Evidence of Factorial Validity and Precision). Revista Colombiana de Psicología, 27(1), 27-40. https://doi.org/10.15446/rcp.v27n1.64467

Halverson, E. R. (2005). InsideOut: Facilitating gay youth identity development through a performance-based youth organization. Identity, 5(1), 67-90. https://doi.org/10.1207/s1532706xid0501_5

Halverson, E. R. (2010). Film as identity exploration: A multimodal analysis of youth-produced films. Teachers College Record, 112 (9), 2352-2378. Retrieved from https://www.tcrecord.org/Content.asp?ContentId=15948

Hemetsberger, A. (2003). When Consumers Produce on the Internet: The Relationship Between Cognitive-affective, Socially-based, and Behavioral Involvement of Prosumers. The Journal of Social Psychology, 12, 1-20.

Hughes, D. J., Rowe, M., Batey, M., \& Lee, A. (2012). A tale of two sites: Twitter vs. Facebook and the personality predictors of social media usage. Computers in Human Behavior, 28(2), 561-569. https://doi.org/10.1016/j.chb.2011.11.001

Igartua, J.-J. \& Rodríguez-de-Dios, I. (2020). Correlatos motivacionales del uso y la satisfacción con Facebook en jóvenes españoles (Motivational correlates of use and satisfaction with Facebook). Cuadernos.Info, (38), 107-119. https://doi.org/10.7764/cdi.38.848

Josselson, R. (1980). Ego development in adolescence. In J. Adelson (Ed.), Handbook of adolescent psychology (pp. 188-210). New York, NY: Wiley. 
Jung, E. H. \& Sundar, S. S. (2018). Status update: Gratifications derived from Facebook affordances by older adults. New Media \& Society, 20(11), 4135-4154. https://doi.org/10.1177/1461444818768090

Krallman, A., Pelletier, M. J., \& Adams, F. G. (2016). @ Size vs.\# Impact: Social media engagement differences amongst Facebook, Twitter, and Instagram. In K. Kim (Ed.), Celebrating America's pastimes: Baseball, hot dogs, apple pie and marketing? (pp. 557-561). Springer.

Litt, E. (2012). Knock, knock. Who's there? The imagined audience. Journal of Broadcasting $\mathcal{E}$ Electronic Media, 56(3), 330-345. https://doi.org/10.1080/08838151.2012.705195

Livingstone, S. (2004). Media literacy and the challenge of new information and communication technologies. The Communication Review, 7(1), 3-14.https://doi.org/10.1080/10714420490280152

Manago, A. M., Graham, M. B., Greenfield, P. M., \& Salimkhan, G. (2008). Self-presentation and gender on MySpace. Journal of Applied Developmental Psychology, 29(6), 446-458. https://doi.org/10.1016/j.appdev.2008.07.001

Marcia, J. E. (1966). Development and validation of ego identity status. Journal of Personality and Social Psychology, 3(5), 551-558. https://doi.org/10.1037/h0023281

Martinez, R. O. \& Dukes, R. L. (1997). The effects of ethnic identity, ethnicity, and gender on adolescent well-being. Journal of Youth and Adolescence, 26(5), 503-516. https://doi.org/10.1023/A:1024525821078

Martínez-Sala, A., Segarra-Saavedra, J., \& Monserrat-Gauchi, J. (2018). Los millennials como prosumers y adprosumers en las redes sociales corporativas (Millennials as prosumers and adprosumers in corporate social networks). Cuadernos.Info, (43), 137-159. https://doi.org/10.7764/cdi.43.1335

Nagy, P. \& Koles, B. (2014). The digital transformation of human identity: Towards a conceptual model of virtual identity in virtual worlds. Convergence, 20(3), 276-292. https://doi.org/10.1177/1354856514531532

Namey, E., Guest, G., Thairu, L., \& Johnson, L. (2007). Data reduction techniques for large qualitative data sets. In G. Guest \& K. MacQueen (Eds.), Handbook for team-based qualitative research (pp. 137-161). Lanham, MD: Altamira Press.

Napoli, P. M. (2014). Automated media: An institutional theory perspective on algorithmic media production and consumption. Communication Theory, 24(3), 340-360. https://doi.org/10.1111/comt.12039

Nunes, F. \& Mota, C. P. (2018). Parenting Styles and Dimensions Questionnaire - Adaptação da Versão Portuguesa de Heterorrelato. Revista Colombiana de Psicología, 27(1), 117-131. https://doi.org/10.15446/rcp.v27n1.64621

Pempek, T. A., Yermolayeva, Y. A., \& Calvert, S. L. (2009). College students' social networking experiences on Facebook. Journal of Applied Developmental Psychology, 30(3), 227-238. https://doi.org/10.1016/j.appdev.2008.12.010

Queluz, F. N. F. R., Campos, C. R. F., Santis, L., Isaac, L., \& Barham, E. J. (2019). Zarit Caregiver Burden Interview: Evidências de Validade para a População Brasileira de Cuidadores de Idosos (The Zarit Caregiver Burden Interview: Evidence of Validity in a Brazilian Population of Senior Citizen Caregivers). Revista Colombiana de Psicología, 28(1), 99-113. https://doi.org/10.15446/rcp.v28n1.69422 
Ross, C., Orr, E. S., Sisic, M., Arseneault, J. M., Simmering, M. G., \& Orr, R. R. (2009). Personality and motivations associated with Facebook use. Computers in Human Behavior, 25(2), 578-586. https://doi.org/10.1016/j.chb.2008.12.024

Serrano-Puche, J. (2013). Vidas conectadas: tecnología digital, interacción social e identidad (Connected lives: digital technology, social interaction and identity). Historia $y$ Comunicación Social, 18, 353-364. https://doi.org/10.5209/rev_HICS.2013.v18.44249

Seidman, G. (2013). Self-presentation and belonging on Facebook: How personality influences social media use and motivations. Personality and Individual Differences, 54(3), 402-407. https://doi.org/10.1016/j.paid.2012.10.009

Schoen-Ferreira, T. H., Aznar-Farias, M., \& Silvares, E. F. D. M. (2003). A construção da identidade em adolescentes: um estudo exploratório (Adolescent's identity construction: an exploratory study). Estudos de Psicologia, 8(1), 107-115. https://doi.org/10.1590/S1413-294X2003000100012

Sinclair, T. J. \& Grieve, R. (2017). Facebook as a source of social connectedness in older adults. Computers in Human Behavior, 66, 363-369. https://doi.org/10.1016/j.chb.2016.10.003

Winter, C. (2012). How media prosumers contribute to social innovation in today's new networked music culture and economy. International Journal of Music Business Research, 1(2), 46-73.

Zappavigna, M. (2014). Enacting identity in microblogging through ambient affiliation. Discourse E Communication, 8(2), 209-228. https://doi.org/10.1177/1750481313510816

\section{SOBRE LOS AUTORES}

JAVIER ALEJANDRO CORREDOR-ARISTIZÁBAL, Profesor asociado del departamento de Psicología en la Universidad Nacional de Colombia. Director actual del grupo de Cognición, Prácticas y Aprendizaje. Su investigación se enfoca en la influencia de la experiencia en ambientes digitales, incluyendo procesos de aprendizaje, educación, desarrollo de la identidad, y construcción de espacios colaborativos de aprendizaje. Doctor en Estudios Cognitivos en Educación de la Universidad de Pittsburgh.

iD http://orcid.org/0000-0003-0893-6332

MANUEL ALBERTO CORREDOR-ARISTIZÁBAL, Docente Facultad de la Facultad de Arte, Comunicación y Cultura, Departamentos de Comunicación Social y Cine y Televisión de la Fundación Universitaria Agustiniana - Uniagustiniana Colombia. Ha sido director de programación del Canal Universitario Nacional (Zoom) y del Sistema de Medios Públicos, Canal Señal Colombia. Ha realizado diversas investigaciones sobre televisión cultural, industrias creativas e innovación digital, publicadas en diversas revistas internacionales. Es Magíster en Innovación del Instituto Tecnológico y de Estudios Superiores de Monterrey, México. 